\title{
Periodic Wave Patterns of two-dimensional Boussinesq systems
}

\author{
Min Chen ${ }^{1}$ and Gérard Iooss ${ }^{2}$ \\ ${ }^{1}$ Department of Mathematics, Purdue University, \\ West Lafayette, IN 47907, USA \\ ${ }^{2}$ Institut Universitaire de France, INLN UMR 6618 CNRS-UNSA \\ 1361 route des Lucioles, F-06560 Valbonne, France
}

August 28, 2005

\begin{abstract}
We prove the existence of a large family of two-dimensional travelling wave patterns for a Boussinesq system which describes three-dimensional water waves. This model equations result from full Euler equations in assuming that the depth of the fluid layer is small with respect to the horizontal wave length, and that the flow is potential, with a free surface without surface tension. Our proof uses Lyapunov-Schmidt method which may be managed here, contrary to the case of gravity waves with full Euler equations. Our results are in a good agreement with experimental results.
\end{abstract}

\section{Introduction}

To describe small-amplitude and long wavelength (the depth is small with respect to wave length) gravity waves of an ideal, incompressible liquid, the system

$$
\begin{aligned}
& \eta_{t}+\nabla \cdot \mathbf{v}+\nabla \cdot(\eta \mathbf{v})-\frac{1}{6} \Delta \eta_{t}=0 \\
& \mathbf{v}_{t}+\nabla \eta+\frac{1}{2} \nabla|\mathbf{v}|^{2}-\frac{1}{6} \Delta \mathbf{v}_{t}=0
\end{aligned}
$$

was put forward by Bona, Chen and Saut [1], where $\eta(\mathbf{x}, t)$ and $\mathbf{v}(\mathbf{x}, t)$, scaled by $h_{0}$ and $\sqrt{g h_{0}}$ respectively with $g$ being the acceleration of gravity and $h_{0}$ being the average water depth, represent the dimensionless deviation of the water surface from its undisturbed position and the horizontal velocity at the level of $\sqrt{2 / 3} h_{0}$ of the depth of the undisturbed fluid, respectively.

The study of two-dimensional wave patterns has been evolved around the KP equation since KP equation admits such solutions explicitly, assuming that the nonlinear waves are nearly one-dimensional. Genuinely, two-dimensional nonlinear waves are observed experimentally (that is three-dimensional waves with 
two-dimensional surface) outside the putative range of validity of the KP equation [8]. Since the system (1) is derived without the KP assumptions that the wave is nearly one-dimensional and predominantly in one-direction, the traveling two-dimensional wave patterns of system (1) are genuinely two-dimensional. The existence of such wave patterns is proved using the bifurcation theory with the bifurcation parameter being the phase velocity. To our knowledge, for the full Euler equations, there are existence results for doubly periodic horizontally two-dimensional travelling waves, only in presence of surface tension (see for example $[9,3,7,6])$. In the case of no surface tension, one can find extensive numerical results (in putting the surface tension equal to 0) reported in [2]. Our result on system (1) which has no surface tension agrees qualitatively with the results in [2] in the appropriate parameter region. We note here that system (1) can be modified, such as adding a term $-\tau \nabla \Delta \eta$ or $\tau \Delta \mathbf{v}_{t}$ on the left-hand side of the second equation, where $\tau=\Gamma / \rho g h_{0}^{2}$ is the Bond number with $\Gamma$ being the surface tension coefficient and $\rho$ being the density of water, to describe waves with surface tension effects [5]. It shows that the transition from no surface tension to small surface tension is smooth, which would justify the use of no surface tension models for water waves.

We have restricted our study to the two-dimensional wave patterns which are symmetric about the direction of the propagation. For other possible choices, see the discussion in [4]. One consequence of our results is that for almost any $(k, \tau)$, where the wave lengths in the horizontal directions are $2 \pi / k$ and $2 \pi /(k \tau)$ respectively, and for small enough wave height, there is a doubly periodic wave pattern with a transport velocity which is slightly above $c_{0}=36\left(1+\tau^{2}\right) /(6+$ $\left.k^{2}\left(1+\tau^{2}\right)\right)^{2}$.

It is worth to note that the problem we consider here is easier than the problem with the full Euler equations, because the operators occurring here are explicit, contrary to the Dirichlet-Neumann operator which is implicit, and because the dispersion equation is nicer in the sense that except few bad choices of the critical parameter, we can use the Lyapunov-Schmidt method, contrary to the full Euler equations where small divisors occur, in absence of surface tension (see discussion in [4]).

Structure of the paper: we set the problem of two-dimensional periodic travelling waves for the system (1) in section 2 . In section 3 we study the linearization near the 0 equilibrium solution, discussing extensively the consequences of the structure of the dispersion relation, which allows to define a smoothing pseudo-inverse of the linearized operator (see theorem 1). The bifurcation analysis is made at section 4 , in taking into account the symmetries of the problem. The main result is Theorem 2 which gives the existence and the form of bifurcating doubly periodic waves in function of the velocity of the waves, and of the angle between the two basic symmetric mono-periodic travelling waves, which are nonlinearly superposed here. Finally we give the shape of the waves in 6 cases for different values of the angle between the basic mono-periodic waves. The qualitative agreement with experiments for small values of this angle is clear (experiments with large angles do not exist yet). 


\section{Position of the problem}

We are looking for solutions of system (1) under the form of 2-dimensional travelling waves, i.e. $\eta$ and $\mathbf{v}$ are periodic functions of $\mathbf{x}-\mathbf{c t}$, where $\mathbf{x}=$ $\left(x_{1}, x_{2}\right) \in \mathbb{R}^{2}$, and $\mathbf{c}$ is the velocity of the travelling wave, which plays the role of a parameter. The periodicity is both in $x_{1}$ and $x_{2}$. For these solutions, the system (1) reads as

$$
\begin{gathered}
\nabla \cdot(\mathbf{v}+\eta \mathbf{v})-\mathbf{c} \cdot \nabla\left(\eta-\frac{1}{6} \Delta \eta\right)=0 \\
\nabla\left(\eta+\frac{1}{2}|\mathbf{v}|^{2}\right)-\mathbf{c} \cdot \nabla\left(\mathbf{v}-\frac{1}{6} \Delta \mathbf{v}\right)=0
\end{gathered}
$$

A first observation is that the subspace where $\operatorname{curl}(\mathbf{v})=0$ is invariant under (2). This comes from the fact that the original system (1) leads to

$$
\partial_{t}\left\{\operatorname{curl}\left(\mathbf{v}-\frac{1}{6} \Delta \mathbf{v}\right)\right\}=0
$$

which implies for an irrotational initial condition and due to the double periodicity of $\mathbf{v}$, that $\operatorname{curl}(\mathbf{v})=0$ holds for any $t$.

Indeed, system (2) has a first integral, coming from the identity

$$
\nabla\left\{\eta+\frac{1}{2}|\mathbf{v}|^{2}-\mathbf{c} \cdot\left(\mathbf{v}-\frac{1}{6} \Delta \mathbf{v}\right)\right\} \cdot \mathbf{c}=0
$$

from which we deduce that

$$
\eta+\frac{1}{2}|\mathbf{v}|^{2}-\mathbf{c} \cdot\left(\mathbf{v}-\frac{1}{6} \Delta \mathbf{v}\right)=\beta\left(x_{2}\right)
$$

where $\beta$ is an arbitrary function of the coordinate $x_{2}$ orthogonal to the direction $x_{1}$ of the travelling wave. Moreover, if this function $\beta$ is a constant, then, from the second equation of (2) we obtain

$$
\operatorname{curl}\left(\mathbf{v}-\frac{1}{6} \Delta \mathbf{v}\right)=0
$$

and then, because of periodicity, $\operatorname{curl}(\mathbf{v})=0$. Conversely, $\operatorname{curl}(\mathbf{v})=0$ in $(2)$ implies that the function $\beta$ is a constant. Since the system (1) was derived in assuming the 3-dimensional flow to be potential, this implies that our twodimensional vector field $\mathbf{v}$ satisfies

$$
\operatorname{curl}(\mathbf{v})=0
$$

So, we do not restrict the analysis in assuming from now-on that the 2-dimensional flow is potential. It is clear that we might eliminate $\eta$ from the system (2), and derive a unique scalar equation for the velocity potential. We prefer to stay with the above formulation (2), since the operators are simpler, and next computations are more transparent. 


\section{Study of the linearized operator}

We start the study by an examination of the linearized problem, that we denote by

$$
\mathcal{L}_{c} U=\mathcal{G} F
$$

where

$$
\begin{gathered}
U=(\eta, \mathbf{v}), \quad F=(g, \mathbf{f}) \\
\mathcal{L}_{c} U=\left(\begin{array}{c}
\nabla \cdot \mathbf{v}-\mathbf{c} \cdot \nabla\left(\eta-\frac{1}{6} \Delta \eta\right) \\
\nabla \eta-\mathbf{c} \cdot \nabla\left(\mathbf{v}-\frac{1}{6} \Delta \mathbf{v}\right)
\end{array}\right), \\
\mathcal{G} F=(\nabla \cdot \mathbf{f}, \nabla g) .
\end{gathered}
$$

It is clear that if $U=(\eta, \mathbf{v})$ is such that $\operatorname{curl}(\mathbf{v})=0$, then $\mathcal{L}_{c} U$ possesses the same property. Moreover, for any $F=(g, \mathbf{f})$, the vector $\mathcal{G} F$ possesses the curl free property for its two last components.

The vector function $\mathbf{f}$ and scalar function $g$ are periodic with Fourier series

$$
\begin{aligned}
\mathbf{f}(\mathbf{x}, t) & =\sum_{\mathbf{k} \in \Gamma^{\prime}} \mathbf{f}_{\mathbf{k}} e^{i \mathbf{k} \cdot \mathbf{x}} \\
g(\mathbf{x}, t) & =\sum_{\mathbf{k} \in \Gamma^{\prime}} g_{\mathbf{k}} e^{i \mathbf{k} \cdot \mathbf{x}}
\end{aligned}
$$

and we look for periodic solutions $(\eta, \mathbf{v})$ such that $\operatorname{curl}(\mathbf{v})=0$, with the Fourier series

$$
\begin{aligned}
\eta(\mathbf{x}, t) & =\sum_{\mathbf{k} \in \Gamma^{\prime}} \eta_{\mathbf{k}} e^{i \mathbf{k} \cdot \mathbf{x}} \\
\mathbf{v}(\mathbf{x}, t) & =\sum_{\mathbf{k} \in \Gamma^{\prime}} \mathbf{v}_{\mathbf{k}} e^{i \mathbf{k} \cdot \mathbf{x}}
\end{aligned}
$$

where $\Gamma^{\prime}$ is a lattice of the plane defined by two vectors $\mathbf{K}_{1}, \mathbf{K}_{2}$, and

$$
\mathbf{k} \in \boldsymbol{\Gamma}^{\prime}: \mathbf{k}=\left(k_{1}, k_{2}\right)=n_{1} \mathbf{K}_{1}+n_{2} \mathbf{K}_{2}, \quad n_{1}, n_{2} \in \mathbb{Z} .
$$

We assume later that the two vectors $\mathbf{K}_{1}$ and $\mathbf{K}_{2}$ are symmetric with respect to the $x_{1}$ axis, making an angle $\pm \theta$ with this axis which will be the direction of c. Then we get for $\mathbf{k} \in \Gamma^{\prime}$

$$
\begin{aligned}
-\left(1+\frac{1}{6}|\mathbf{k}|^{2}\right)(\mathbf{c} \cdot \mathbf{k}) \eta_{\mathbf{k}}+\mathbf{k} \cdot \mathbf{v}_{\mathbf{k}} & =\mathbf{k} \cdot \mathbf{f}_{\mathbf{k}} \\
\mathbf{k} \eta_{\mathbf{k}}-\left(1+\frac{1}{6}|\mathbf{k}|^{2}\right)(\mathbf{c} \cdot \mathbf{k}) \mathbf{v}_{\mathbf{k}} & =\mathbf{k} g_{\mathbf{k}}
\end{aligned}
$$

Let us define

$$
\Delta(\mathbf{k}, \mathbf{c})=\left(1+\frac{1}{6}|\mathbf{k}|^{2}\right)^{2}(\mathbf{c} \cdot \mathbf{k})^{2}-|\mathbf{k}|^{2}
$$

The linearized operator has a non trivial kernel if there exists a pair $\left(\mathbf{k}_{0}, \mathbf{c}_{0}\right)$ satisfying

$$
\Delta\left(\mathbf{k}_{0}, \mathbf{c}_{0}\right)=0
$$


or if $\left(\mathbf{k}_{0} \cdot \mathbf{c}_{0}\right)=0$. We need to study the set of $\mathbf{k}$ in the plane, satisfying $\Delta(\mathbf{k}, \mathbf{c})=0$ for a given velocity $\mathbf{c}$, to obtain the kernel of the linearized operator. The big restriction is that our vectors $\mathbf{k}$ need to belong to the lattice $\Gamma^{\prime}$.

If $\Delta \neq 0$ and $(\mathbf{k} \cdot \mathbf{c}) \neq 0$, we obtain

$$
\begin{aligned}
& \eta_{\mathbf{k}}=-\frac{\left(1+\frac{1}{6}|\mathbf{k}|^{2}\right)(\mathbf{c} \cdot \mathbf{k})\left(\mathbf{k} \cdot \mathbf{f}_{\mathbf{k}}\right)+|\mathbf{k}|^{2} g_{\mathbf{k}}}{\Delta(\mathbf{k}, \mathbf{c})}, \\
& \mathbf{v}_{\mathbf{k}}=-\mathbf{k} \frac{\left(1+\frac{1}{6}|\mathbf{k}|^{2}\right)(\mathbf{c} \cdot \mathbf{k}) g_{\mathbf{k}}+\mathbf{k} \cdot \mathbf{f}_{\mathbf{k}}}{\Delta(\mathbf{k}, \mathbf{c})},
\end{aligned}
$$

where we notice that

$$
\operatorname{curl}\left(\mathbf{v}_{k} e^{i \mathbf{k} \cdot \mathbf{x}}\right)=0,
$$

while for $(\mathbf{k} \cdot \mathbf{c})=0$, we have, $\mathbf{k}=\left(0, k_{2}\right)$ and

$$
\begin{aligned}
\eta_{\mathbf{k}} & =g_{\mathbf{k}}, \\
\left(v_{1}\right)_{\mathbf{k}} & =\text { const } \\
\left(v_{2}\right)_{\mathbf{k}} & =\left(f_{2}\right)_{\mathbf{k}}
\end{aligned}
$$

where the constant value of $\left(v_{1}\right)_{\mathbf{k}}$ is necessary for satisfying the condition $\operatorname{curl}\left(\mathbf{v}_{k} e^{i k_{2} x_{2}}\right)=0$. We give below estimates for $\left(\eta_{\mathbf{k}}, \mathbf{v}_{\mathbf{k}}\right)$ in terms of $\left(\mathbf{f}_{\mathbf{k}}, g_{\mathbf{k}}\right)$ in cases of $\Delta(\mathbf{k}, \mathbf{c}) \neq 0$. These estimates allow to give a bound of the pseudoinverse of the linearized operator on its range.

We first study the set of wave vectors $\mathbf{k}$ such that $\Delta(\mathbf{k}, \mathbf{c})=0$. Assume $\mathbf{c}=(c, 0)$, and let $\mathbf{k}=\left(k_{1}, k_{2}\right)=n_{1} \mathbf{K}_{1}+n_{2} \mathbf{K}_{2}$ satisfies $\Delta(\mathbf{k}, \mathbf{c})=0$, where

$$
\begin{aligned}
\mathbf{K}_{1} & =k(1, \tau), \quad \mathbf{K}_{2}=k(1,-\tau) \\
k & >0, \quad c>0
\end{aligned}
$$

which means that $\tau=\tan \theta, \pm \theta$ being the angles between the axis $x_{1}$ and the wave vectors $\mathbf{K}_{1}$, and $\mathbf{K}_{2}$. We then have

$$
\begin{aligned}
k_{1} & =m_{1} k, \quad k_{2}=m_{2} \tau k \\
m_{1} & =n_{1}+n_{2} \\
m_{2} & =n_{1}-n_{2},
\end{aligned}
$$

and we observe that $m_{1}$ and $m_{2}$ have the same parity. Replacing in the equation $\Delta(\mathbf{k}, \mathbf{c})=0$ we obtain

$$
\left(m_{1} c\right)^{2}\left\{1+\frac{k^{2}}{6}\left(m_{1}^{2}+\tau^{2} m_{2}^{2}\right)\right\}^{2}=m_{1}^{2}+\tau^{2} m_{2}^{2}
$$

We observe for a given $(c, k, \tau)$ that for any solution $\left(m_{1}, m_{2}\right)$ of $(11)$ this corresponds in fact to the 4 solutions

$$
\left( \pm m_{1}, \pm m_{2}\right)
$$


Let $(c, k, \tau)=\left(c_{0}, k, \tau\right)$ be such that we have a solution $\left(m_{1}, m_{2}\right)=(1,1)$ of (11). This means that

$$
c_{0}^{2}\left\{1+\frac{k^{2}}{6}\left(1+\tau^{2}\right)\right\}^{2}=1+\tau^{2}
$$

First observe that for $m_{1}=0$, equation (11) cannot be satisfied for $m_{2} \neq 0$. Now for $m_{1} \neq 0$, equation (11) can only have a finite number of solutions, since for any solution $\left(m_{1}, m_{2}\right)$ we have (study the maximum of the function $\frac{x}{\left(1+\frac{k^{2}}{6} x\right)^{2}}$ for $x \in(0, \infty))$

$$
m_{1}^{2} \leq \frac{6}{4 c_{0}^{2} k^{2}}
$$

and when $m_{1}$ is fixed, the quantity $m_{1}^{2}+\tau^{2} m_{2}^{2}$ is the root of a second degree polynomial, with the additional condition for $m_{2}$ to be an integer.

It is then clear that in general $\left(m_{1}, m_{2}\right)=(1,1)$ is the only solution in $\mathbb{N}^{2}$ of (11). Consider now six examples of such choices for $\left(c_{0}, k, \tau\right)$. Indeed, let us choose $k=\frac{1}{2}$, which corresponds to fix the wave length in $x_{1}$ as $4 \pi$ (this is large with respect to 1 , which is one condition to respect for the model system (1) to be valid. Then we have

$$
c_{0}^{2}=\frac{1+\tau^{2}}{\left\{1+\frac{1}{24}\left(1+\tau^{2}\right)\right\}^{2}} \leq 6
$$

where $\tau=\tan \theta \in(0,+\infty)$. Let consider the following typical cases:

i) $\tau=1 / 10, c_{0}=\frac{240 \sqrt{101}}{2501}\left(\theta=5.71^{\circ}\right)$,

ii) $\tau=2-\sqrt{3}, c_{0}=\frac{6 \sqrt{2}}{61}(7 \sqrt{3}-5) \quad\left(\theta=\pi / 12=15^{\circ}\right)$,

iii) $\tau=1 / \sqrt{3}, c_{0}=\frac{12 \sqrt{3}}{19} \quad\left(\theta=\pi / 6=30^{\circ}\right)$,

iv) $\tau^{2}=1, c_{0}=\frac{12 \sqrt{2}}{13} \quad\left(\theta=\pi / 4=45^{\circ}\right)$.

v) $\tau^{2}=3, c_{0}=\frac{12}{7} \quad\left(\theta=\pi / 3=60^{\circ}\right)$,

vi) $\tau=3, c_{0}=\frac{12 \sqrt{10}}{17} \quad\left(\theta=71.6^{\circ}\right)$,

It is easy, to show, using (12) and the expression (11) for $m_{1}=1$ and 2 , that the solution $\left(m_{1}, m_{2}\right)=(1,1)$ is the only solution in $\mathbb{N}^{2}$ of $(11)$ for all the above particular cases.

Assuming more generally that $\left(c_{0}, k, \tau\right)$ is in the situation where $\left(m_{1}, m_{2}\right)=$ $(1,1)$ is the only solution in $\mathbb{N}^{2}$ of $(11)$, we see that for $m_{1} \neq 0$, and $\left|m_{1}\right|+\left|m_{2}\right|>$ $M$, we have the following minoration of $\Delta$ :

$$
|\Delta|>C_{1} m_{1}^{2}\left(m_{1}^{2}+\tau^{2} m_{2}^{2}\right)^{2}
$$

where $C_{1}$ is independent of $\left(m_{1}, m_{2}\right)$, which leads to

$$
\left|\eta_{\mathbf{k}}\right|+|| \mathbf{v}_{\mathbf{k}}||<C_{2} \frac{\left|g_{\mathbf{k}}\right|+|| \mathbf{f}_{\mathbf{k}}||}{m_{1}\left(\left|m_{1}\right|+\left|m_{2}\right|\right)},
$$


with $C_{2}$ independent of $\left(m_{1}, m_{2}\right)$. Moreover, we have by hypothesis for $\left(m_{1}, m_{2}\right) \neq$ $(0,0)$ and $( \pm 1, \pm 1),\left|m_{1}\right|+\left|m_{2}\right| \leq M, m_{1} \neq 0$,

$$
\left|\eta_{\mathbf{k}}\right|+|| \mathbf{v}_{\mathbf{k}}||<C_{3}\left(\left|g_{\mathbf{k}}\right|+|| \mathbf{f}_{\mathbf{k}}||\right) .
$$

Finally, for $m_{1}=0$, we choose to take $\left(v_{1}\right)_{\mathbf{k}}=0$ (see (10)), and the above estimate is trivially satisfied.

We conclude that for $\left(m_{1}, m_{2}\right) \neq(0,0)$ and $( \pm 1, \pm 1)$, and for our choice of determination of $v_{1}$ in the case when $m_{1}=0$,

$$
\left|\eta_{\mathbf{k}}\right|+|| \mathbf{v}_{\mathbf{k}}||<C\left(\left|g_{\mathbf{k}}\right|+|| \mathbf{f}_{\mathbf{k}}||\right) .
$$

It remains to study the case when $\left(m_{1}, m_{2}\right)=(0,0)$ or $( \pm 1, \pm 1)$.

For $\left(m_{1}, m_{2}\right)=(0,0)$ we find easily that $\eta_{\mathbf{0}}$ and $\mathbf{v}_{\mathbf{0}}$ are arbitrary, hence giving a subspace of dimension 3 (this subspace has indeed a one-dimensional intersection with the part of the kernel given for $\left.m_{1}=0\right)$.

For $\left(m_{1}, m_{2}\right)=( \pm 1, \pm 1)$, i.e. for $\mathbf{k}=k( \pm 1, \pm \tau)$, we have for $(g, \mathbf{f})$ the compatibility condition under the form

$$
|\mathbf{k}| g_{\mathbf{k}}+\operatorname{sgn}\left(m_{1}\right) \mathbf{k} \cdot \mathbf{f}_{\mathbf{k}}=0
$$

i.e. for instance, for $\mathbf{k}=k(1, \tau)$, i.e. $\left(m_{1}, m_{2}\right)=(1,1)$

$$
\sqrt{1+\tau^{2}} g_{1,1}+\left(\mathbf{f}_{1}\right)_{1,1}+\tau\left(\mathbf{f}_{2}\right)_{1,1}=0 .
$$

When the condition (13) is realized, then

$$
\begin{aligned}
\eta_{\mathbf{k}} & =g_{\mathbf{k}}+\beta \sqrt{1+\tau^{2}} \\
\mathbf{v}_{\mathbf{k}} & =\beta\left(1, \tau \operatorname{sgn}\left(m_{1}\right) \operatorname{sgn}\left(m_{2}\right)\right)
\end{aligned}
$$

where $\beta$ is arbitrary in $\mathbb{C}$. For $\mathbf{k}=k\left(m_{1}, \tau m_{2}\right)$, where $\left(m_{1}, m_{2}\right)=( \pm 1, \pm 1)$, let us define the eigenvectors $\xi_{\mathbf{k}}$ by

$$
\xi_{\mathbf{k}}=\left(\sqrt{1+\tau^{2}}, 1, \tau \operatorname{sgn}\left(m_{1}\right) \operatorname{sgn}\left(m_{2}\right)\right) e^{i k\left(m_{1} x_{1}+\tau m_{2} x_{2}\right)},
$$

then we have by construction

$$
\mathcal{L}_{c_{0}} \xi_{\mathbf{k}}=0
$$

and with the hermitian scalar product in $\left\{L_{\text {如 }}^{2}\right\}^{3}$, the compatibility condition (13) reads

$$
\left\langle F, \xi_{\mathbf{k}}\right\rangle=0, \quad \mathbf{k}=k( \pm 1, \pm \tau) .
$$

Now defining the Sobolev space

$$
H_{\text {如 }}^{l}=\left\{u=\sum_{\mathbf{k} \in \Gamma^{\prime}} u_{k} e^{i \mathbf{k x}} \in H^{l}\{(\mathbb{R} /(2 \pi / k) \mathbb{Z}) \times(\mathbb{R} /(2 \pi / k \tau) \mathbb{Z})\}\right.
$$

where $\mathbf{k}=k\left(m_{1}, \tau m_{2}\right), m_{1}, m_{2} \in \mathbb{Z}, m_{1}$ and $m_{2}$ have same parity $\}$. 
The condition on the parities of $m_{1}$ and $m_{2}$ comes from the construction (recall that $\left.m_{1}=n_{1}+n_{2}, m_{2}=n_{1}-n_{2}\right)$. This leads to the invariance under the shift

$$
\sigma:\left(x_{1}, x_{2}\right) \mapsto\left(x_{1}+\frac{\pi}{k}, x_{2}+\frac{\pi}{k \tau}\right) .
$$

The above calculations mean that we are able to define a bounded operator $\mathcal{T}$ acting in $\left\{H_{\text {如 }}^{p}\right\}^{3} \cap\{\operatorname{curl}(\mathbf{v})=0\}$, for any $p \geq 0$, solving (4), provided the compatibility condition (13) is satisfied, and such that

$$
\begin{aligned}
U & =\mathcal{T} F \\
\mathcal{T} F_{\mathbf{k}} & =U_{\mathbf{k}}=\left(\eta_{\mathbf{k}}, \mathbf{v}_{\mathbf{k}}\right),
\end{aligned}
$$

is given by (8), (9) for $\Delta \neq 0$ and ( $\mathbf{k} \cdot \mathbf{c}) \neq 0$, i.e. for $\mathbf{k} \neq k( \pm 1, \pm \tau)$, and for $k_{1}=k m_{1} \neq 0$. For $k_{1}=0, k_{2} \neq 0$

$$
\mathcal{T} F_{\mathbf{k}}=\left(g_{\mathbf{k}}, 0,\left(f_{2}\right)_{\mathbf{k}}\right),
$$

and for $\mathbf{k}=(0,0)$

$$
\mathcal{T} F_{0}=0,
$$

while for $\mathbf{k}=k( \pm 1, \pm \tau)$ we set

$$
\mathcal{T} F_{\mathbf{k}}=\left(g_{\mathbf{k}}, \mathbf{0}\right) .
$$

Notice that the operator $\mathcal{T}$ is defined here, even for $F=(g, \mathbf{f})$ not satisfying the compatibility condition (14).

Since we restrict our attention to solutions symmetric with respect to the axis $x_{1}$ of propagation, let us introduce the new spaces

$$
H_{\text {㛎 }}^{l, e}=\left\{w \in H_{\text {如 }}^{l} ; w \text { is even in } x_{2}\right\}
$$

and similarly $H_{\text {如 }}^{l, o}=\left\{w \in H_{\text {如 }}^{l}, w\right.$ is odd in $\left.x_{2}\right\}$. Then, the above linear operator $\mathcal{T}$ acts from $E_{p}=\left(H_{\text {始 }}^{p, e}\right)^{2} \times\left(H_{\text {蜡 }}^{p, o}\right)$ into

$$
G_{p}=\left(H_{\text {蛇 }}^{p, e}\right)^{2} \times\left(H_{\text {蜡 }}^{p, o}\right) \cap\{\operatorname{curl}(\mathbf{v})=0\},
$$

and (2) can be understood in this space for $p \geq 2$ (this restriction is for having the nonlinear terms $\left.\left(\frac{\mathbf{v}^{2}}{2}, \eta \mathbf{v}\right) \in\left(H_{\natural \natural}^{p, e}\right)^{2} \times\left(H_{\natural \natural}^{p, o}\right)\right)$. In the following we use the following notations:

$$
\begin{aligned}
\xi_{0} & =\frac{1}{2}\left(\xi_{(1,1)}+\xi_{(1,-1)}\right) \\
\bar{\xi}_{0} & =\frac{1}{2}\left(\xi_{(-1,1)}+\xi_{(-1,-1)}\right),
\end{aligned}
$$

i.e.

$$
\xi_{0}=e^{i k x_{1}}\left(\sqrt{1+\tau^{2}} \cos k \tau x_{2}, \cos k \tau x_{2}, i \tau \sin k \tau x_{2}\right),
$$


and $\xi_{0} \in G_{p}$ for any $p$. Notice that any $F \in E_{p}, p>0$, has a Fourier expansion of the form

$$
\begin{aligned}
F & =\sum_{\mathbf{m} \in \mathbb{Z} \times \mathbb{N}_{0}} F_{\mathbf{m}} e^{i k m_{1} x_{1}}, \\
F_{\mathbf{m}} & =\left(g_{\mathbf{m}} \cos k \tau m_{2} x_{2},\left(\mathbf{f}_{1}\right)_{\mathbf{m}} \cos k \tau m_{2} x_{2}, i\left(\mathbf{f}_{2}\right)_{\mathbf{m}} \sin k \tau m_{2} x_{2}\right),
\end{aligned}
$$

where $m_{1}$ and $m_{2}$ have the same parity in the sum, and $\mathbb{N}_{0}=\mathbb{N} \cup\{0\}$. It then results for instance that the 4 components of the compatibility condition (14) reduces to the two conditions

$$
\left\langle F, \xi_{0}\right\rangle=0, \quad\left\langle F, \bar{\xi}_{0}\right\rangle=0 .
$$

Now, let us introduce the $O(2)$ group invariance, corresponding to the invariance of the system under shifts in $x_{1}$ and reflection $x_{1} \mapsto-x_{1}$. The operator representing the shift is $\tau_{a}$ defined by

$$
\left(\tau_{a} U\right)\left(x_{1}, x_{2}\right)=U\left(x_{1}+a, x_{2}\right)
$$

and the symmetry operator $S$ is defined by

$$
S U\left(x_{1}, x_{2}\right)=\left(\eta\left(-x_{1}, x_{2}\right), \mathbf{v}_{1}\left(-x_{1}, x_{2}\right),-\mathbf{v}_{2}\left(-x_{1}, x_{2}\right)\right) .
$$

It is then clear that the $O(2)$ symmetry is satisfied since

$$
\tau_{\frac{2 \pi}{k}}=\mathbb{I}, \quad S \tau_{a}=\tau_{-a} S
$$

and the systems (2) and (4) commute with $S$ and with $\tau_{a}$, for any $a$ real. Then, we can assert the following

Theorem 1. Assume that $\left(c_{0}, k, \tau\right)$ is such that $\left(m_{1}, m_{2}\right)=(1,1)$ is the only solution in $\mathbb{N}^{2}$ of (11). Then, for any given

$$
F=(g, \mathbf{f}) \in E_{p}=\left(H_{\text {如 }}^{p, e}\right)^{2} \times\left(H_{\text {如 }}^{p, o}\right), \quad p \geq 0,
$$

such that the following compatibility conditions

$$
\left\langle F, \xi_{0}\right\rangle=\left\langle F, \bar{\xi}_{0}\right\rangle=0
$$

hold for the Fourier components with $\mathbf{k}=k( \pm 1, \pm \tau)$, the general solution $U=$ $(\eta, \mathbf{v}) \in G_{p}$ of the system

$$
\mathcal{L}_{c_{0}} U=\mathcal{G F}
$$

is given by

$$
U=\mathcal{T} F+A \xi_{0}+\overline{A \xi}_{0}+C \zeta_{0}+D \zeta_{1},
$$

where

$$
\begin{aligned}
& \zeta_{0}=(1,0,0), \quad \zeta_{1}=(0,1,0) \\
& \xi_{0}=\left(\sqrt{1+\tau^{2}} \cos k \tau x_{2}, \cos k \tau x_{2}, i \tau \sin k \tau x_{2}\right) e^{i k x_{1}}
\end{aligned}
$$


$A \in \mathbb{C}, C, D \in \mathbb{R}$, and $\mathcal{T}$ is the bounded linear operator defined above. Moreover, we have the symmetry properties

$$
\begin{aligned}
S \mathcal{T} F & =\mathcal{T} S F, \tau_{a} \mathcal{T} F=\mathcal{T} \tau_{a} F \\
S \zeta_{0} & =\tau_{a} \zeta_{0}=\zeta_{0}, \\
S \zeta_{1} & =\tau_{a} \zeta_{1}=\zeta_{1}, \\
S \xi_{0} & =\bar{\xi}_{0}, \tau_{a} \xi_{0}=e^{i k a} \xi_{0} .
\end{aligned}
$$

Remark: The existence of eigenvectors $\zeta_{1}$ and $\zeta_{0}$ for the linear operator $\mathcal{L}_{c}$ comes from the important property of system (2) to possess the family of "trivial" solutions

$$
U^{(0)}=(C, D, 0)
$$

for arbitrary constants $C, D$.

\section{Bifurcating solutions}

Let us set $c=\frac{c_{0}}{1+\mu}$, and rewrite the equation as

$$
\mathcal{L}_{c_{0}} U+\mu \mathcal{G} U+(1+\mu) \mathcal{G} B(U, U)=0,
$$

where

$$
B(U, U)=\left(\frac{1}{2}|\mathbf{v}|^{2}, \eta \mathbf{v}\right) .
$$

Then we decompose $U \in G_{p}$ as

$$
U=A \xi_{0}+\overline{A \xi}_{0}+C \zeta_{0}+D \zeta_{1}+V,
$$

where $V$ has the same properties as $\mathcal{T} F$ above, i.e.

$$
V_{0}=0
$$

for $\mathbf{k}=k( \pm 1, \pm \tau)$

$$
V_{\mathbf{k}}=\left(\eta_{\mathbf{k}}, \mathbf{0}\right),
$$

for $k_{1}=0, k_{2} \neq 0$

$$
V_{\mathbf{k}}=\left(\eta_{\mathbf{k}}, 0,\left(\mathbf{v}_{2}\right)_{\mathbf{k}}\right) .
$$

Notice that with our definition of $V$, we have

$$
\begin{aligned}
\left\langle V, \zeta_{1}\right\rangle & =\left\langle V, \zeta_{0}\right\rangle=0, \\
\left\langle V, \xi_{0}\right\rangle & =\sqrt{1+\tau^{2}} \frac{2 \pi^{2}}{k^{2} \tau} \eta_{(1,1)} .
\end{aligned}
$$

Replacing the expression for $U$ in the nonlinear equation (17), the unknown are $V, A, C, D$ in function of the parameter $\mu$. The form of (17) implies, from Theorem 1, that we can rewrite equation (17) as

$$
\begin{aligned}
V+\mu \mathcal{T} U+(1+\mu) \mathcal{T} B(U, U) & =0, \\
\mu A\left\langle\xi_{0}, \xi_{0}\right\rangle+\mu\left\langle V, \xi_{0}\right\rangle+(1+\mu)\left\langle B(U, U), \xi_{0}\right\rangle & =0,
\end{aligned}
$$


where the second equation (compatibility condition) is in $\mathbb{C}$, the writing of the complex conjugate equation of (19) being useless. Notice that the first equation may be solved by the implicit function theorem in $G_{p}$ with respect to $V$, and that by construction we have

$$
\begin{aligned}
\mathcal{T} \zeta_{0} & =\mathcal{T} \zeta_{1}=\mathbf{0} \\
\mathcal{T} \xi_{0} & =\left(\sqrt{1+\tau^{2}} \cos k \tau x_{2} e^{i k x_{1}}, \mathbf{0}\right) .
\end{aligned}
$$

It then results that for $A, C, D, \mu$, close enough to 0 , we obtain

$$
V=\mathcal{V}(A, \bar{A}, C, D, \mu) \in G_{p}
$$

and, because of the $O(2)$ invariance and the uniqueness of $\mathcal{V}$, the following property holds:

$$
\begin{aligned}
\tau_{a} \mathcal{V}(A, \bar{A}, C, D, \mu) & =\mathcal{V}\left(A e^{i k a}, \bar{A} e^{-i k a}, C, D, \mu\right) \\
S \mathcal{V}(A, \bar{A}, C, D, \mu) & =\mathcal{V}(\bar{A}, A, C, D, \mu)
\end{aligned}
$$

A consequence of these symmetry properties is that if $A \equiv 0$, then $\mathcal{V}$ and $X$ are invariant under shifts $\tau_{a}$, which means that $U$ is independent of $x_{1}$. It then results, because of the parity in $x_{2}$ that $U=U^{(0)}$ lies in the family of "trivial solutions" mentioned above.A careful examination of the principal part of $\mathcal{V}$ shows that

$$
\mathcal{V}(A, \bar{A}, C, D, \mu)=-\mu A \mathcal{T} \xi_{0}-\mu \bar{A} \mathcal{T} \bar{\xi}_{0}-\mathcal{T} B(X, X)+O\left(\mid \mu\|\| X\left\|^{2}+\right\| X \|^{3}\right),
$$

with

$$
\begin{aligned}
& X=A \xi_{0}+\overline{A \xi}_{0}+C \zeta_{0}+D \zeta_{1}, \\
& B(X, X)=\left(\begin{array}{c}
\frac{1}{2} D^{2}+D \cos k \tau x_{2}\left(A e^{i k x_{1}}+\bar{A} e^{-i k x_{1}}\right) \\
+\left(\frac{A^{2}}{4} e^{2 i k x_{1}}+\frac{\bar{A}^{2}}{4} e^{-2 i k x_{1}}\right)\left(1-\tau^{2}+\left(1+\tau^{2}\right) \cos 2 k \tau x_{2}\right) \\
+\frac{|A|^{2}}{2}\left(1+\tau^{2}+\left(1-\tau^{2}\right) \cos 2 k \tau x_{2}\right) \\
C D+\left(C+D \sqrt{1+\tau^{2}}\right) \cos k \tau x_{2}\left(A e^{i k x_{1}}+\bar{A} e^{-i k x_{1}}\right) \\
+|A|^{2} \sqrt{1+\tau^{2}}\left(1+\cos 2 k \tau x_{2}\right)+ \\
+\frac{\sqrt{1+\tau^{2}}}{2}\left(A^{2} e^{2 i k x_{1}}+\bar{A}^{2} e^{-2 i k x_{1}}\right)\left(1+\cos 2 k \tau x_{2}\right) \\
i \tau C \sin k \tau x_{2}\left(A e^{i k x_{1}}-\bar{A} e^{-i k x_{1}}\right) \\
+\frac{i \tau \sqrt{1+\tau^{2}}}{2} \sin 2 k \tau x_{2}\left(A^{2} e^{2 i k x_{1}}-\bar{A}^{2} e^{-2 i k x_{1}}\right)
\end{array}\right) .
\end{aligned}
$$

With simple, but tedious computations, and by denoting

$$
U=X-V_{1}-V_{2}+O\left(|\mu|\|X\|^{2}+\|X\|^{3}\right)
$$

where

$$
\begin{aligned}
V_{1} & =\mu A \mathcal{T} \xi_{0}+\mu \bar{A} \mathcal{T} \bar{\xi}_{0} \\
V_{2} & =\mathcal{T} B(X, X)
\end{aligned}
$$


we obtain

$$
\begin{gathered}
V_{1}=\mu \sqrt{1+\tau^{2}}\left(A e^{i k x_{1}}+\bar{A} e^{-i k x_{1}}\right) \cos \left(k \tau x_{2}\right)(1,0,0) \\
V_{2}=|A|^{2} \zeta_{2}+\zeta_{3} D\left(A e^{i k x_{1}}+\bar{A} e^{-i k x_{1}}\right)+ \\
+\left(A^{2} e^{2 i k x_{1}}+\bar{A}^{2} e^{-2 i k x_{1}}\right) \xi_{1}+A^{2} e^{2 i k x_{1}} \xi_{2}+\bar{A}^{2} e^{-2 i k x_{1}} \bar{\xi}_{2},
\end{gathered}
$$

where

$$
\begin{gathered}
\zeta_{2}=\left(\frac{1}{2}\left(1-\tau^{2}\right) \cos 2 k \tau x_{2}, 0,0\right) \\
\zeta_{3}=\left(\cos k \tau x_{2}, 0,0\right) \\
\xi_{1}=\left(c_{1}, c_{2}, 0\right) \\
\xi_{2}=\left(d_{1} \cos 2 k \tau x_{2}, d_{2} \cos 2 k \tau x_{2}, i \tau d_{2} \sin 2 k \tau x_{2}\right), \\
c_{1}=\frac{2\left(1+\frac{2}{3} k^{2}\right) c_{0} \sqrt{1+\tau^{2}}+1-\tau^{2}}{4-4\left(1+\frac{2}{3} k^{2}\right)^{2} c_{0}^{2}} \\
c_{2}=\frac{\left(1+\frac{2}{3} k^{2}\right) c_{0}\left(1-\tau^{2}\right)+2 \sqrt{1+\tau^{2}}}{4-4\left(1+\frac{2}{3} k^{2}\right)^{2} c_{0}^{2}} \\
d_{1}=\frac{\left\{c_{0} \sqrt{1+\tau^{2}}\left(1+\frac{2}{3} k^{2}\left(1+\tau^{2}\right)\right)+\frac{1}{2}\left(1+\tau^{2}\right)\right\}\left(1+\tau^{2}\right)}{2\left(1+\tau^{2}\right)-2 c_{0}^{2}\left(1+\frac{2}{3} k^{2}\left(1+\tau^{2}\right)\right)^{2}} \\
d_{2}=\frac{\left\{\frac{c_{0}}{2}\left(1+\frac{2}{3} k^{2}\left(1+\tau^{2}\right)\right)+\sqrt{1+\tau^{2}}\right\}\left(1+\tau^{2}\right)}{2\left(1+\tau^{2}\right)-2 c_{0}^{2}\left(1+\frac{2}{3} k^{2}\left(1+\tau^{2}\right)\right)^{2}} .
\end{gathered}
$$

Now replacing $V$ by $\mathcal{V}$ in (19), we obtain one equation in $\mathbb{C}$ of the form

$$
h(A, \bar{A}, C, D, \mu)=0
$$

such that

$$
\begin{aligned}
h\left(A e^{i k a}, \bar{A} e^{-i k a}, C, D, \mu\right) & =e^{i k a} h(A, \bar{A}, C, D, \mu) \\
h(A, \bar{A}, C, D, \mu) & =\bar{h}(\bar{A}, A, C, D, \mu)
\end{aligned}
$$

holds for any real $a$, because of the $O(2)$ equivariance of the system (18), (19). It results that $h$ takes the following form

$$
h(A, \bar{A}, C, D, \mu)=A Q\left(|A|^{2}, C, D, \mu\right) .
$$


We observe that $2 B\left(U_{1}, U_{2}\right)=\left(\mathbf{v}_{1} \cdot \mathbf{v}_{2}, \eta_{1} \mathbf{v}_{2}+\eta_{2} \mathbf{v}_{1}\right)$ and

$$
\begin{aligned}
\left\langle B(X, X), \xi_{0}\right\rangle= & \frac{2 \pi^{2}}{k^{2} \tau}\left\{2 \sqrt{1+\tau^{2}} D+\left(1+\tau^{2}\right) C\right\} A, \\
\mu\left\langle\mathcal{V}, \xi_{0}\right\rangle= & -\frac{2 \pi^{2}}{k^{2} \tau} \sqrt{1+\tau^{2}}\left\{\mu^{2} \sqrt{1+\tau^{2}}+\mu D\right\} A \\
& +O\left(\mu^{2}\left\|\left.X\right|^{2}+|\mu||| X\right\|^{3}\right), \\
\left\langle 2 B\left(A \xi_{0},-|A|^{2} \zeta_{2}\right), \xi_{0}\right\rangle= & -\frac{2 \pi^{2}}{k^{2} \tau} A|A|^{2} \frac{1}{4}\left(1-\tau^{2}\right)^{2}, \\
\left\langle 2 B\left(\overline{A \xi}_{0},-A^{2} e^{2 i k x_{1}} \xi_{1}\right), \xi_{0}\right\rangle= & -\frac{2 \pi^{2}}{k^{2} \tau} A|A|^{2}\left\{2 \sqrt{1+\tau^{2}} c_{2}+\left(1-\tau^{2}\right) c_{1}\right\}, \\
\left\langle 2 B\left(\overline{A \xi}_{0},-A^{2} e^{2 i k x_{1}} \xi_{2}\right), \xi_{0}\right\rangle= & -\frac{2 \pi^{2}}{k^{2} \tau} A|A|^{2}\left\{\left(1+\tau^{2}\right)^{3 / 2} d_{2}+\frac{1+\tau^{2}}{2} d_{1}\right\},
\end{aligned}
$$

hence

$$
\begin{aligned}
Q & =2 \mu\left(1+\tau^{2}\right)+2 \sqrt{1+\tau^{2}} D+\left(1+\tau^{2}\right) C+ \\
& -|A|^{2}\left(2 \sqrt{1+\tau^{2}} c_{2}+\left(1-\tau^{2}\right) c_{1}+\frac{1}{4}\left(1-\tau^{2}\right)^{2}+\left(1+\tau^{2}\right)^{3 / 2} d_{2}+\frac{1+\tau^{2}}{2} d_{1}\right)+ \\
& +O\left\{\left(|\mu|+|C|+|D|+|A|^{2}\right)^{2}\right\} .
\end{aligned}
$$

An easy consequence is that $A=0$ is a solution of our problem. This corresponds to $X=C \zeta_{0}+D \zeta_{1}$, and finally to the family (16) of trivial solutions $U^{(0)}$.

Non trivial solutions are given by the solutions of the real equation

$$
Q\left(|A|^{2}, C, D, \mu\right)=0 .
$$

At principal order this leads to

$$
|A|^{2}=\frac{1}{d}\left\{2 \mu\left(1+\tau^{2}\right)+2 \sqrt{1+\tau^{2}} D+\left(1+\tau^{2}\right) C\right\}+O\left\{(|\mu|+|C|+|D|)^{2}\right\}
$$

where

$$
d=2 \sqrt{1+\tau^{2}} c_{2}+\left(1-\tau^{2}\right) c_{1}+\frac{1}{4}\left(1-\tau^{2}\right)^{2}+\left(1+\tau^{2}\right)^{3 / 2} d_{2}+\frac{1+\tau^{2}}{2} d_{1}
$$

provided that $d \neq 0$. Indeed $d$ is a non constant meromorphic function of $\tau$ in a neighborhood of the positive real axis, hence it may cancel only for a discrete set of isolated values of $\tau \in(0, \infty)$. Finally we proved the following

Theorem 2. Assume that $\left(c_{0}, k, \tau\right)$ is such that $\left(m_{1}, m_{2}\right)=(1,1)$ is the only solution in $\mathbb{N}^{2}$ of (11), then for any $p \geq 2$, there is a neighborhood of $\left(c_{0}, 0,0\right)$ in $\mathbb{R}^{3}$ such that the bifurcation surface for $\left(c_{b}, C, D\right)$, where $c_{b}$ is the critical velocity of the travelling wave, $C$ and $D$ are respectively the averages of the elevation $\eta$ and the horizontal velocity along the wave, is analytic and satisfies

$$
\frac{c_{b}}{c_{0}}=1+\frac{D}{\sqrt{1+\tau^{2}}}+\frac{C}{2}+O\left\{(|C|+|D|)^{2}\right\}
$$


The bifurcation of travelling waves of the form $U=(\eta, \mathbf{v}) \in G_{p}$ takes place for $c>c_{b}$ if the coefficient $d$ defined in (24) is $<0$, and for $c<c_{b}$ if $d>0$, the amplitude is such that $|A|^{2}$ is analytic in $\left(c-c_{b}, C, D\right)$, satisfying

$$
|A|^{2}=\left(c_{b}-c\right)\left\{\frac{2\left(1+\tau^{2}\right)}{c_{0} d}+O\left(\left|c_{b}-c\right|+|C|+|D|\right)\right\} .
$$

Moreover

$$
\begin{aligned}
U & =A \xi_{0}+\overline{A \xi}_{0}+C \zeta_{0}+D \zeta_{1}+\mathcal{V}(A, \bar{A}, C, D, \mu) \\
\mathcal{V}(A, \bar{A}, C, D) & =O\left\{|A|\left(|\mu|+|D|+|A|+|C|^{2}\right)\right\}
\end{aligned}
$$

where $\mu=\frac{c_{0}}{c}-1$, and a phase shift $\phi$ on $A$ corresponds to a translation $\phi / k$ for $x_{1}$.

Corollary 3. With the assumptions of the theorem above, the form of the free surface, even in $x_{1}$, and with $C=D=0$, is given at leading order by

$\eta=\varepsilon \cos k x_{1} \cos k \tau x_{2}-\frac{\varepsilon^{2}}{2\left(1+\tau^{2}\right)}\left\{\frac{1-\tau^{2}}{4} \cos 2 k \tau x_{2}+c_{1} \cos 2 k x_{1}+d_{1} \cos 2 k x_{1} \cos 2 k \tau x_{2}\right\}+O\left(\varepsilon^{3}\right)$

where $\varepsilon=2|A| \sqrt{1+\tau^{2}}$. For $\tau$ close to 0, we have

$\eta \approx \varepsilon \cos k x_{1} \cos k \tau x_{2}-\varepsilon^{2}\left\{\frac{1}{8} \cos 2 k \tau x_{2}+c_{1}^{(0)} \cos 2 k x_{1}+c_{1}^{(0)} \cos 2 k x_{1} \cos 2 k \tau x_{2}\right\}+O\left(\varepsilon^{3}\right)$

with

$$
c_{1}^{(0)}=\frac{1+2 c_{0}^{(0)}\left(1+\frac{2}{3} k^{2}\right)}{4-4\left(c_{0}^{(0)}\right)^{2}\left(1+\frac{2}{3} k^{2}\right)^{2}}, \quad c_{0}^{(0)}=\frac{1}{1+\frac{k^{2}}{6}},
$$

and

$$
\begin{gathered}
\varepsilon^{2} \approx \frac{8\left(c_{b}-c\right)}{c_{0}^{(0)} d^{(0)}}, \\
d^{(0)}=\frac{6 c_{0}^{(0)}\left(1+\frac{2}{3} k^{2}\right)+\frac{15}{2}}{4-4\left(c_{0}^{(0)}\right)^{2}\left(1+\frac{2}{3} k^{2}\right)^{2}}+\frac{1}{4}<0 \text { for } k \text { small enough. }
\end{gathered}
$$

Remark 1: we notice that for $\tau$ close to 0 , there are "nodal lines" given by $x_{2}=\pi / 2 k \tau$ modulo $(\pi / k \tau)$, since, up to order $\varepsilon^{2}$ included, we have $\eta \approx \frac{\varepsilon^{2}}{8}$ which is independent of $x_{1}$.

Remark 2: It is seen experimentally that for moderate values of $\varepsilon$ it appears a local maximum in the troughs (between to strong minima). This is an effect easily explained with the influence of order $\varepsilon^{2}$ terms. The occurrence of local minima between two strong maxima, only occurs in presence of order $\varepsilon^{3}$ terms.

The function $\eta\left(x_{1}, x_{2}\right)$ is computed numerically for the six special cases mentioned earlier. The corresponding angle between the basic wave vectors $2 \theta$ range from $11.4^{\circ}$ to $143^{\circ}$. We take $k=\frac{1}{2}$ and $\varepsilon=0.2$ in our computation to make sure that the wavelength in the $x_{1}$ direction $L_{x_{1}}=2 \pi / k>>1$, the 

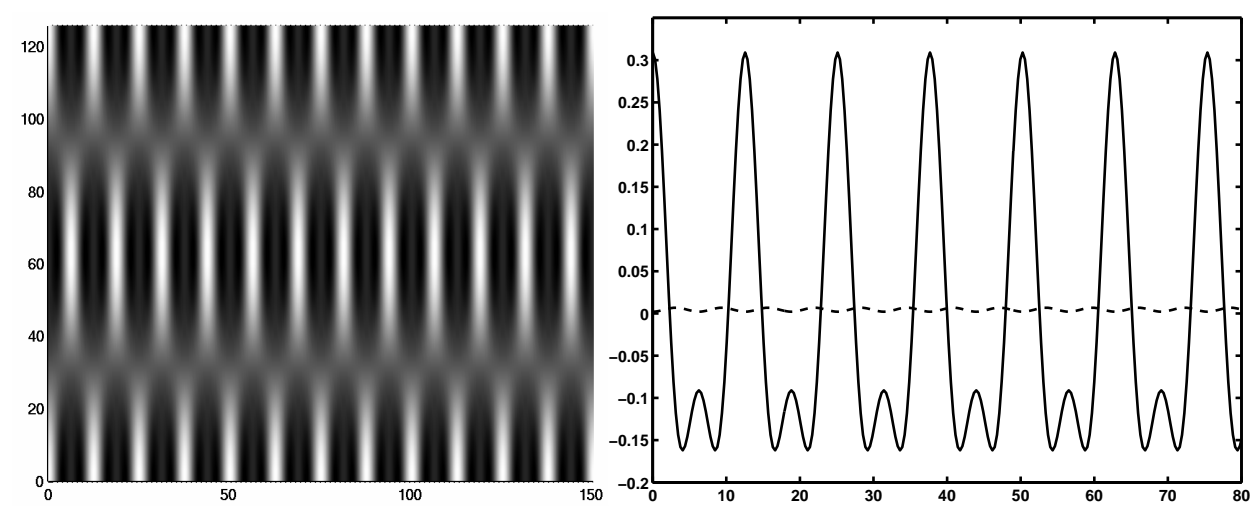

Figure 1: $\tau=1 / 10, L_{x_{1}}=12.6, L_{x_{2}}=126, c_{0}=0.96, c \approx 1.03$

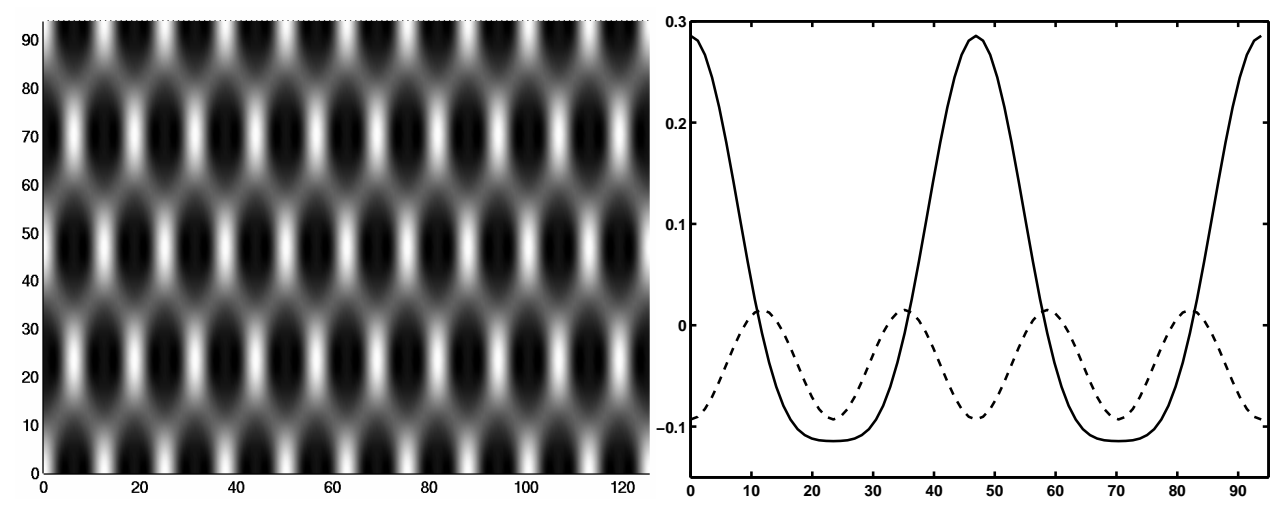

Figure 2: $\tau=2-\sqrt{3}, L_{x_{1}}=12.6, L_{x_{2}}=46.96, c_{0}=0.99, c \approx 1.04$

wavelength in the $x_{2}$ direction $L_{x_{2}}=2 \pi /(k \tau)>>1$ and the wave height is small (we recall that the still water depth is scaled to 1 in our model). The transport velocity $c=c_{0}\left(1-\frac{d \varepsilon^{2}}{8\left(1+\tau^{2}\right)^{2}}\right)+$ higher order terms.

In Figure 1, we take $\tau=1 / 10$ which is in the KP range. The surface profile $\eta\left(x_{1}, x_{2}\right)$ is plotted on the left and the surface profiles at $x_{2}=0$ (solid line) and at $x_{2}=L_{x_{2}} / 4=\pi /(2 k \tau)$ (dashed line) are plotted on the right. We first note that the surface seems to comprise of rectangular cells, with nodal lines at $x_{2}=L_{x_{2}} / 4=\pi /(2 k \tau)$ (see the dashed line on the right), which is a phenomena often linked with deep water models. Furthermore, there is a local maximum in the valley region.

In Figure 2, we take $\tau=2-\sqrt{3}$. The surface profiles $\eta\left(x_{1}, x_{2}\right)$ is plotted on the left and the surface profiles at $x_{1}=0$ (solid line) and at $x_{1}=L_{x_{1}} / 4=\pi /(2 k)$ (dashed line) are plotted on the right. The surface clear comprise of hexagonal cells with no nodal lines. In the $x_{2}$ direction, the crest is narrower than the valley (see the solid line). 

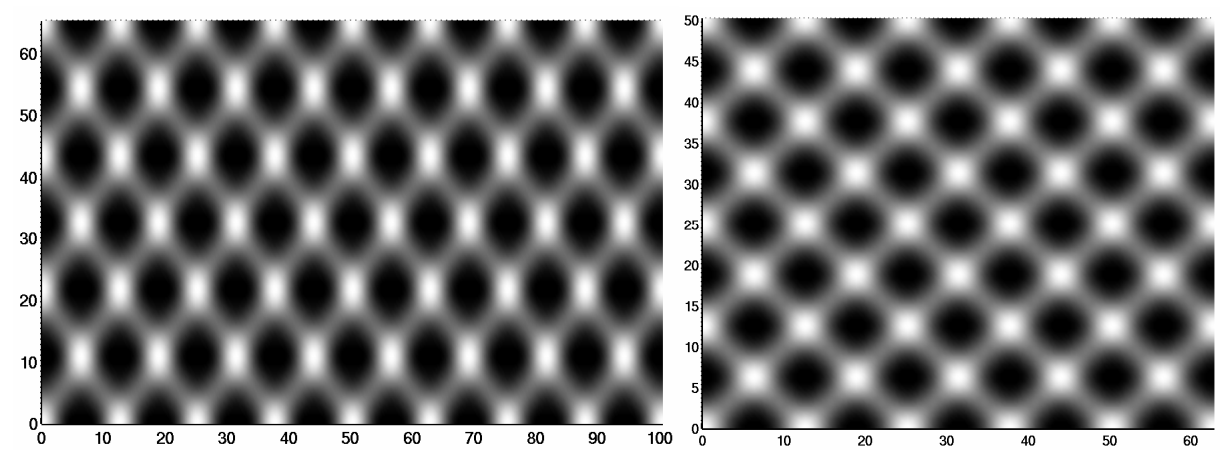

Figure 3: $\tau=1 / \sqrt{3}$ and $\tau=1$
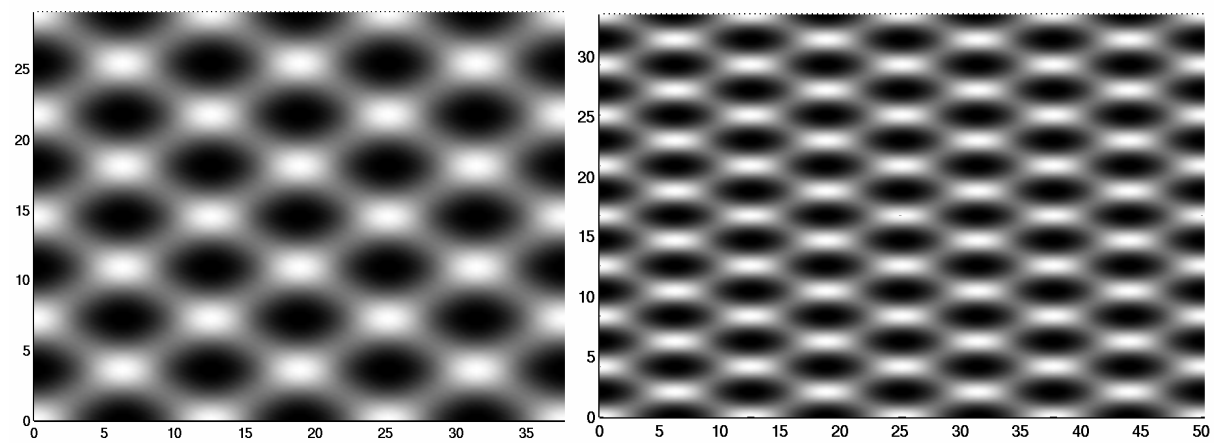

Figure 4: $\tau=\sqrt{3}$ and $\tau=3$

The surface profiles for $\tau=1 / \sqrt{3}$ and $\tau=1$ are plotted in Figure 3. Since our model does not require the wave to be weakly two-dimensional, it does not require $\tau$ to be small as for the KP equation. For $\tau=1 / \sqrt{3}$ the figure shows hexagonal cells, while for $\tau=1$ there are square cells. However, the solution has not such symmetries. In the latter case, the invariance under a rotation by $\pi / 2$ is broken at the $\left(\varepsilon^{2}\right)$ order.

On Figure 4 we plot surface profiles for $\tau=\sqrt{3}$ and $\tau=3$. The last surface resembles the surface in Figure 2 rotated by $90^{\circ}$.

\section{References}

[1] J. L. Bona, M. Chen, And J.-C. SAut, Boussinesq equations and other systems for small-amplitude long waves in nonlinear dispersive media I: Derivation and the linear theory, J. Nonlinear Sci., 12 (2002), pp. 283-318.

[2] T. J. Bridges, F. Dias, And D. Menasce, Steady three-dimensional water-wave patterns on a finite-depth fluid, J. Fluid Mech., 436 (2001), 
pp. $145-175$.

[3] W. Craig and D. P. Nicholls, Travelling two and three dimensional capillary gravity water waves, SIAM J. Math. Anal., 32 (2000), pp. 323-359 (electronic).

[4] - Traveling gravity water waves in two and three dimensions, Eur. J. Mech. B Fluids, 21 (2002), pp. 615-641.

[5] P. DARIPA And R. K. DAsh, A class of model equations for bi-directional propagation of capillary-gravity waves, Internat. J. Engrg. Sci., 41 (2003), pp. 201-218.

[6] M. D. Groves AND M. Haragus, A bifurcation theory for threedimensional oblique travelling gravity-capillary water waves, J. Nonlinear Sci., 13 (2003), pp. 397-447.

[7] M. D. Groves And A. Mielke, A spatial dynamics approach to threedimensional gravity-capillary steady water waves, Proc. Roy. Soc. Edinburgh Sect. A, 131 (2001), pp. 83-136.

[8] J. Hammack, N. Scheffner, and H. Segur, Two-dimensional periodic waves in shallow water, J. Fluid Mech., 209 (1989), pp. 567-589.

[9] J. ReEder AND M. Shinbrot, Three-dimensional, nonlinear wave interaction in water of constant depth, Nonlinear Anal., 5 (1981), pp. 303-323. 